\title{
GENERATING FUNCTIONS ASSOCIATED TO FROBENIUS ALGEBRAS
}

\author{
JOSEP ÀLVAREZ MONTANER
}

\begin{abstract}
We introduce a generating function associated to the homogeneous generators of a graded algebra that measures how far is this algebra from being finitely generated. For the case of some algebras of Frobenius endomorphisms we describe this generating function explicitly as a rational function.
\end{abstract}

\section{INTRODUCTION}

Let $R$ be a commutative Noetherian ring and let $A=\oplus_{e \geq 0} A_{e}$ be a $\mathbb{N}$-graded ring, such that $A_{0}=R$ so it has a natural structure as $R$-algebra. Although $A$ may not necessarily be commutative, we will assume for simplicity that $A$ is a left skew $R$-algebra, that is $a R \subseteq R a$ for all homogeneous elements $a \in A$.

In the case that $A$ is not a finitely generated $R$-algebra it is natural to ask how far is this algebra from being finitely generated by measuring the number of generators that we have at each degree. Under these premises, F. Enescu and Y. Yao introduced in [6] the complexity sequence of $A$, where the integer components $c_{e}$ of this sequence measure the number of homogeneous generators of degree $e$ that can not be obtained from homogeneous elements of lower degree. More precisely:

Definition 1.1. Let $A=\oplus_{e \geq 0} A_{e}$ be a $\mathbb{N}$-graded ring. Set $G_{-1}=A_{0}$ and let $G_{e}(A)=G_{e}$ be the subring of $A$ generated by the elements of degree $\leq e$. Let $c_{e}:=c_{e}(A)$ be the number of homogeneous generators of $A_{e} /\left(G_{e-1}(A)\right)_{e}$ over $A_{0}$. Then, the complexity sequence of $A$ is $\left\{c_{e}(A)\right\}_{e \geq 0}$.

From now on, we will only consider the case of degree-wise finitely generated $\mathbb{N}$-graded algebras ensuring that $c_{e}<+\infty$ for all $e$. The asymptotic behaviour of this sequence allowed F. Enescu and Y. Yao to introduce a new invariant, the complexity of $A$.

Definition 1.2. Let $\left\{c_{e}(A)\right\}_{e \geq 0}$ be the complexity sequence of a $\mathbb{N}$-graded $R$-algebra $A$. The complexity of $A$ is

$$
\operatorname{cx}(A):=\inf \left\{\lambda \in \mathbb{R}_{>0} \mid c_{e}(A)=O\left(\lambda^{e}\right)\right\} .
$$

If there is no such an $\lambda$ we say that $\operatorname{cx}(A)=\infty$.

2010 Mathematics Subject Classification. Primary 13A02, 13A35 Secondary 39A10.

Key words and phrases. Frobenius algebra, complexity sequence, linear recurrence.

Partially supported by Generalitat de Catalunya 2017 SGR-932 project and Ministerio de Economía y Competitividad MTM2015-69135-P. He is also with the Barcelona Graduate School of Mathematics (BGSMath). 
A motivating example is when $R$ is a local complete commutative ring of positive characteristic $p>0$ and $A=\mathcal{F}\left(E_{R}\right)$ is the Frobenius algebra associated to the injective hull of the residue field, that we denote as $E_{R}$. This is a non-commutative $\mathbb{N}$-graded $R$-algebra introduced by G. Lyubeznik and K. E. Smith in [13] that collects all possible Frobenius actions on $E_{R}$. The study of these algebras has its roots in the theory of tight closure introduced by M. Hochster and C. Huneke [9]. The dual notion of Cartier algebra (see [16], [3]) plays a prominent role in the theory of singularities in positive characteristic.

For the case of Frobenius algebras, F. Enescu and Y. Yao coined in [6] the notion of Frobenius complexity. Its interest comes from the fact that, for some particular examples, the limit of this invariant as $p \rightarrow \infty$ exists so it may be interpreted as an invariant of $R$ in characteristic zero.

Definition 1.3. Let $R$ be a local complete commutative ring of positive characteristic $p>0$. The Frobenius complexity of $R$ is $\operatorname{cx}_{F}(R):=\log _{p} \operatorname{cx}\left(\mathcal{F}\left(E_{R}\right)\right)$.

M. Katzman gave in [11] the first example of non-finitely generated Frobenius algebra $\mathcal{F}\left(E_{R}\right)$. Unfortunately, we may not find many examples in the literature where non-finitely generated Frobenius algebras are explicitly described so one may extract the complexity sequence. Among these scarce sources we would like to mention the cases of StanleyReisner rings [1], Veronese subrings [12] or $2 \times 2$ minors of a $2 \times 3$ generic matrix [12]. For the case of $2 \times 2$ minors of a $n \times m$ generic matrix, F. Enescu and Y. Yao [6, 7] gave an indirect approach to compute the complexity sequence. Actually, due to the asymptotic nature of these invariants, one may bound or even compute the Frobenius complexity by comparing with some known cases (see [6, 7]). J. Page [15] also took this indirect approach to compute the Frobenius complexity of the so-called Hibi rings.

In this work, instead of studying the asymptotic behaviour of the complexity sequence, we are more concerned on structural properties of this sequence. In Section 2 we collect the terms of this sequence in a series

$$
\mathcal{G}_{A}(T)=\sum_{e \geq 0} c_{e} T^{e}
$$

that we denote as the generating function of $A$. In the case that $\mathcal{G}_{A}(T)$ is a rational function we obtain an explicit linear recurrence for the coefficients $c_{e}$. This is a very strong condition that implies that the complexity of $A$ is finite since we can read this invariant from the set of poles of the generating function (see Theorem 2.5).

In Section 3 we recall the basics on Frobenius algebras which is the main example we are interested on. Our interest comes from the fact that, for the few examples that we may find in the literature, the corresponding generating function is rational. These computations are developed in Section 4. We pay special attention to the case of determinantal rings treated by F. Enescu and Y. Yao. The main result of this section is Theorem 4.4 where we describe the linear recurrence that the complexity sequence satisfies in this case.

All the computations performed in this paper have been developed using MATLAB [14]. 
Aknowledgements: We greatly appreciate the referee for his/her comments.

\section{Generating FunCtion}

Let $R$ be a commutative Noetherian ring and let $A=\oplus_{e}>_{0} A_{e}$ be a (non-necessarily commutative) $\mathbb{N}$-graded ring. Associated to the complexity sequence of $A$ we may consider the following series:

Definition 2.1. Let $\left\{c_{e}(A)\right\}_{e \geq 0}$ be the complexity sequence of a $\mathbb{N}$-graded algebra $A$. We define the generating function of $A$ as

$$
\mathcal{G}_{A}(T)=\sum_{e \geq 0} c_{e} T^{e}
$$

Remark 2.2. The generating function encodes the same information as the $\mathscr{Z}$-transform of the complexity sequence $\left\{c_{e}(A)\right\}_{e \geq 0}$. Namely, $\mathcal{G}_{A}(T)=\mathscr{Z}\left[c_{e}\right]\left(\frac{1}{T}\right)$ where

$$
\mathscr{Z}\left[c_{e}\right](Z)=\sum_{e \geq 0} \frac{c_{e}}{Z^{e}} .
$$

This alternative approach will be useful in Section 4.3 where we are going to use some elementary properties of the $\mathscr{Z}$-transform without further comment. There is a vast bibliography on the $\mathscr{Z}$-transform, especially in mathematical engineering, so we refer to any textbook such as [5] for more insight.

Remark 2.3. We may define another generating function of $A$ as

$$
\widehat{\mathcal{G}}_{A}(T)=\sum_{e \geq 0} a_{e} T^{e}
$$

where $a_{e}:=a_{e}(A)$ is the number of homogeneous generators of $A_{e}$ over $A_{0}$. The motivation behind considering $\mathcal{G}_{A}(T)$ instead of $\widehat{\mathcal{G}}_{A}(T)$ in this work is because of its relation with the complexity of $A$.

The natural question that arise whenever we have a series as the one defined by the generating function is whether it is a rational function. This property provides a strong structural property of the complexity sequence due to the following well-known result for which we present a sketch of the proof for the sake of completeness.

Proposition 2.4. Let $\left\{c_{e}(A)\right\}_{e \geq 0}$ be the complexity sequence of a $\mathbb{N}$-graded algebra $A$. Let $Q(T)=1+a_{1} T+a_{2} T^{2}+\cdots+a_{k-1} T^{k-1}+a_{k} T^{k}$ be a polynomial of degree $k$ in one variable $T$. Then the following are equivalent:

i) There exists an integer $e_{0} \geq 0$ such that, for all $e \geq e_{0}$, the coefficients of the complexity sequence are given by a linear recurrence relation

$$
c_{e+k}+a_{1} c_{e+k-1}+a_{2} c_{e+k-2}+\cdots+a_{k-1} c_{e+1}+a_{k} c_{e}=0 .
$$


ii) The generating function is a rational function

$$
\mathcal{G}_{A}(T)=c_{0}+c_{1} T+\cdots+T^{e_{0}} \sum_{e \geq e_{0}} c_{e} T^{e-e_{0}}=c_{0}+c_{1} T+\cdots+T^{e_{0}} \frac{P(T)}{Q(T)},
$$

with $\operatorname{deg} P(T)<k$.

Proof. Without loss of generality we may assume $e_{0}=0$. We have:

$$
\begin{aligned}
Q(T) \sum_{e \geq 0} c_{e} T^{e} & =\left(1+a_{1} T+a_{2} T^{2}+\cdots+a_{k-1} T^{k-1}+a_{k} T^{k}\right) \sum_{e \geq 0} c_{e} T^{e}= \\
& =c_{0}+\left(c_{1}+a_{1} c_{0}\right) T+\cdots+\left(c_{k-1}+a_{1} c_{k-2}+\cdots+a_{k-1} c_{0}\right) T^{k-1}+ \\
& +\left(c_{k}+a_{1} c_{k-1}+\cdots+a_{k} c_{0}\right) T^{k}+\left(c_{k+1}+a_{1} c_{k}+\cdots+a_{k} c_{1}\right) T^{k+1}+\cdots
\end{aligned}
$$

Therefore,

$$
c_{e+k}+a_{1} c_{e+k-1}+a_{2} c_{k-2}+\cdots+a_{k-1} c_{e+1}+a_{k} c_{e}=0,
$$

for all $e \geq 0$ if and only if $\mathcal{G}_{A}(T)=\frac{P(T)}{Q(T)}$, with

$$
P(T)=c_{0}+\left(c_{1}+a_{1} c_{0}\right) T+\cdots+\left(c_{k-1}+a_{1} c_{k-2}+\cdots+a_{k-1} c_{0}\right) T^{k-1} .
$$

Theorem 2.5. Assume that the generating function $\mathcal{G}_{A}(T)$ of a $\mathbb{N}$-graded algebra $A$ is a rational function. Then the complexity of $A$ is $\operatorname{cx}(A)=\frac{1}{|\lambda|}$, where $\lambda$ is a pole with minimal absolute value.

Proof. Once again, assume for simplicity, that

$$
\mathcal{G}_{A}(T)=\frac{P(T)}{Q(T)},
$$

with $Q(T)=1+a_{1} T+a_{2} T^{2}+\cdots+a_{k-1} T^{k-1}+a_{k} T^{k}$ and $\operatorname{deg} P(T)<k$. Thus the recurrence relation is

$$
c_{k}+a_{1} c_{k-1}+a_{2} c_{k-2}+\cdots+a_{k-1} c_{1}+a_{k} c_{0}=0 .
$$

In order to solve this linear difference equation, let

$$
q(T)=T^{k}+a_{1} T^{k-1}+a_{2} T^{k-2}+\cdots+a_{k-1} T+a_{k}
$$

be its characteristic polynomial. We have $q(T)=Q\left(\frac{1}{T}\right)$, so the inverses of the roots of $q(T)$ correspond to the poles of $\mathcal{G}_{A}(T)$. Assume that we have a factorization

$$
q(T)=\left(T-\lambda_{1}\right)^{m_{1}}\left(T-\overline{\lambda_{1}}\right)^{m_{1}} \cdots\left(T-\lambda_{r}\right)^{m_{r}}\left(T-\overline{\lambda_{r}}\right)^{m_{r}}\left(T-\lambda_{r+1}\right)^{m_{r+1}} \cdots\left(T-\lambda_{s}\right)^{m_{s}},
$$

with $\lambda_{j}=\left|\lambda_{j}\right| e^{i \Theta_{j}} \in \mathbb{C}$ for $j=1, \ldots, r$ and $\lambda_{j} \in \mathbb{R}$ for $j=r+1, \ldots, s$. Then the elements $c_{e}(A)$ of the complexity sequence are linear combinations of the form 


$$
\begin{aligned}
c_{e}(A) & =\left|\lambda_{1}\right|^{e}\left[\left(\alpha_{1,1}+\alpha_{1,2} e+\cdots+\alpha_{1, m_{1}} e^{m_{1}-1}\right) \cos \Theta_{1}+\left(\alpha_{1,1}^{\prime}+\alpha_{1,2}^{\prime} e+\cdots+\alpha_{1, m_{1}}^{\prime} e^{m_{1}-1}\right) \sin \Theta_{1}\right] \\
\vdots & \\
& +\left|\lambda_{r}\right|^{e}\left[\left(\alpha_{r, 1}+\alpha_{r, 2} e+\cdots+\alpha_{r, m_{r}} e^{m_{r}-1}\right) \cos \Theta_{r}+\left(\alpha_{r, 1}^{\prime}+\alpha_{r, 2}^{\prime} e+\cdots+\alpha_{r, m_{r}}^{\prime} e^{m_{r}-1}\right) \sin \Theta_{r}\right] \\
& +\lambda_{r+1}^{e}\left[\alpha_{r+1,1}+\cdots+\alpha_{r+1, m_{r+1}} e^{m_{r+1}-1}\right]+\cdots+\lambda_{s}^{e}\left[\alpha_{s, 1}+\cdots+\alpha_{s, m_{s}} e^{m_{s}-1}\right] .
\end{aligned}
$$

Notice that each term has order $O\left(\left|\lambda_{i}\right|^{e}\right)$ and thus the order of $c_{e}$ corresponds to the roots of $q(T)$ with maximal absolute value. These roots are the inverse of poles of $\mathcal{G}_{A}(T)$ with minimal absolute value and thus $\operatorname{cx}(A)=\frac{1}{|\lambda|}$ where $\lambda$ is such a pole.

Remark 2.6. The coefficients $\alpha_{i, j}$ and $\alpha_{i, j}^{\prime}$ in the linear combination described above are uniquely determined imposing initial conditions $c_{0}, \ldots, c_{k-1}$. Therefore, the order of the complexity sequence depends on these initial conditions.

Remark 2.7. In all the examples that we will consider, the linear difference equation has a dominant characteristic eigenvalue so their generating function $\mathcal{G}_{A}(T)$ has a unique simple pole with minimum absolute value.

Corollary 2.8. Let $\left\{c_{e}(A)\right\}_{e \geq 0}$ be the complexity sequence of a $\mathbb{N}$-graded algebra $A$ satisfying the linear recurrence relation

$$
c_{e+k}+a_{1} c_{e+k-1}+a_{2} c_{e+k-2}+\cdots+a_{k-1} c_{e+1}+a_{k} c_{e}=0 .
$$

Then, the complexity of $A$ is $\operatorname{cx}(A)=|\lambda|$, if $\lambda$ is a root of the characteristic polynomial

$$
q(T)=T^{k}+a_{1} T^{k-1}+a_{2} T^{k-2}+\cdots+a_{k-1} T+a_{k}
$$

with maximum absolute value.

\section{Frobenius ALGEBRAS}

In this section we will introduce the basics on the theory of Frobenius algebras that we are going to use in the rest of this paper. We point out that one could also consider the dual notion of Cartier algebras so the interested reader should feel free to follow her/his own preference.

Let $R$ be a commutative ring of characteristic $p>0$ and $M$ an $R$-module. We use the $e$-th iterated Frobenius map $F^{e}: R \longrightarrow R$ to define a new $R$-module structure on $M$ given by $r m:=r^{p^{e}} m$ for all $r \in R$ and $m \in M$. One denotes this $R$-module as $F_{*}^{e} M$. Indeed, one may use this to define the $e$-th Frobenius functor from the category of left $R$-modules to itself. In [13], G. Lyubeznik and K. E. Smith introduced the following ring in their study of the localization problem in tight closure. 
Definition 3.1. The ring of Frobenius operators on $M$ is the $\mathbb{N}$-graded, associative, not necessarily commutative ring

$$
\mathcal{F}(M):=\bigoplus_{e \geq 0} \mathcal{F}^{e}(M)
$$

where $\mathcal{F}^{e}(M):=\operatorname{Hom}_{R}\left(M, F_{*}^{e} M\right)$.

The pieces $\mathcal{F}^{e}(M)$ can be identified with the set of all $p^{e}$-linear maps. That is, the set of all additive maps $\varphi_{e}: M \longrightarrow M$ satisfying $\varphi_{e}(r m)=r^{p^{e}} \varphi_{e}(m)$ for all $r \in R, m \in M$. Composing a $p^{e}$-linear map and a $p^{e^{\prime}}$-linear map we get a $p^{\left(e+e^{\prime}\right)}$-linear map so we get a natural ring structure for $\mathcal{F}(M)$. Each $\mathcal{F}^{e}(M)$ is a left module over $\mathcal{F}^{0}(M):=\operatorname{End}_{R}(M)$.

When $(R, \mathfrak{m}, k)$ is a local complete local ring and $E_{R}:=E_{R}(k)$ is the injective hull of the residue field we notice that $\mathcal{F}\left(E_{R}\right)$ is an $R$-algebra due to the fact that

$$
\mathcal{F}^{0}\left(E_{R}\right)=\operatorname{Hom}_{R}\left(E_{R}, E_{R}\right) \cong R \text {. }
$$

Two important instances where the Frobenius algebra can be described more explicitly are presented in the sequel.

3.1. The case of completed $k$-algebras of finite type. Let $S=k\left[\left[x_{1}, \ldots, x_{n}\right]\right]$ be the formal power series ring in $n$ variables over a field $k$ of characteristic $p>0$. Let $I \subseteq S$ be any ideal and $E_{R}$ be the injective hull of the residue field of $R:=S / I$. For this module we have a nice description of the corresponding Frobenius algebra using a result of $\mathrm{R}$. Fedder [8] (see also [2], [10] for more insight). Namely, there exists a natural Frobenius action $F$ from $E_{R}$ onto itself such that for each $e \geq 0$, any $p^{e}$-linear map from $E_{R}$ onto itself is uniquely of the form $g F^{e}$, where $g$ is an element of $\left(I^{\left[p^{e}\right]}:_{S} I\right) / I^{\left[p^{e}\right]}$. So there exists an isomorphism of $R$-modules

$$
\mathcal{F}^{e}\left(E_{R}\right) \cong\left(I^{\left[p^{e}\right]}:_{S} I\right) / I^{\left[p^{e}\right]}
$$

that can be extended in a natural way to an isomorphism of $R$-algebras

$$
\mathcal{F}\left(E_{R}\right) \cong \bigoplus_{e \geq 0}\left(I^{\left[p^{e}\right]}:_{S} I\right) / I^{\left[p^{e}\right]} .
$$

3.2. T-construction. Let $(R, \mathfrak{m}, k)$ be a normal complete local ring of characteristic $p>0$ and $E_{R}$ be the injective hull of the residue field. Let $\omega_{R}$ be a canonical module of $R$. Then, the anticanonical cover of $R$ is the $\mathbb{N}$-graded ring

$$
\mathscr{R}=\bigoplus_{n \geq 0} \omega_{R}^{(-n)}
$$

where $\omega_{R}^{(-n)}$ denotes the $n$-th symbolic power of the divisorial ideal $\omega_{R}^{(-1)}$. M. Katzman, K. Schwede, A. Singh and W. Zhang showed in [12] that the Frobenius algebra $\mathcal{F}\left(E_{R}\right)$ is isomorphic to a subgroup of the anticanonical cover of $R$ with a twisted multiplication that we are going to describe next 
Definition 3.2. Let $\mathscr{R}$ be an $\mathbb{N}$-graded commutative ring of characteristic $p>0$. Associated to this ring, we consider

$$
T(\mathscr{R})=\bigoplus_{e \geq 0} \mathscr{R}_{p^{e}-1}
$$

with a twisted multiplication * on $T(\mathscr{R})$ given by

$$
a * b=a b^{p^{e}} \quad \text { for } a \in T(\mathscr{R})_{e} \text { and } b \in T(\mathscr{R})_{e^{\prime}} .
$$

Using this construction, we get the following interpretation of the Frobenius algebra (see [12, Theorem 3.3] for details).

Theorem 3.3. Let $(R, \mathfrak{m})$ be a normal complete local ring of characteristic $p>0$. Then there exists a graded isomorphism

$$
\mathcal{F}\left(E_{R}\right) \cong T(\mathscr{R})=\bigoplus_{e \geq 0} \omega_{R}^{\left(1-p^{e}\right)}
$$

It follows that $\mathcal{F}\left(E_{R}\right)$ is principal whenever $R$ is Gorenstein. In the case that $R$ is $\mathbb{Q}-$ Gorenstein, combining [12, Proposition 4.1] and [7, Theorem 4.15], we have

Proposition 3.4. Let $(R, \mathfrak{m}, k)$ be a normal complete $\mathbb{Q}$-Gorenstein local ring of characteristic $p>0$. Then

i) $\mathcal{F}\left(E_{R}\right)$ is a finitely generated $R$-algebra if and only if $p$ is relatively prime with the index of $R$.

ii) $\mathcal{F}\left(E_{R}\right)$ is principal if and only if the index of $R$ divides $p-1$.

\section{Generating functions of some Frobenius Algebras}

As we mentioned in the introduction, there are not so many explicit examples of Frobenius algebras that we may find in the literature. In this section we will present them and we will show that the generating function is a rational function, or equivalently, the complexity sequence satisfies a linear recurrence.

4.1. Stanley-Reisner rings. The first example of a non finitely generated Frobenius algebra $\mathcal{F}\left(E_{R}\right)$ was given by M. Katzman in [11] where he considered the (completed) Stanley-Reisner ring $R=k[[x, y, z]] /(x y, y z)$.

More generally, let $S=k\left[\left[x_{1}, \ldots, x_{m}\right]\right]$ be the formal power series ring with coefficients over a field of positive characteristic and let $I$ be a squarefree monomial ideal. For simplicity, we may just consider ideals involving all the variables. A complete description of the Frobenius algebra $\mathcal{F}\left(E_{R}\right)$ associated to the Stanley-Reisner ring $R=S / I$ is given in [1]. Their approach is by means of an explicit computation of the colon ideal $\left(I^{\left[p^{e}\right]}:_{S} I\right)$. It turns out that there are only two possible cases (see [1, Section 3.1] for details)

i) $\left(I^{\left[p^{e}\right]}:_{S} I\right)=I^{\left[p^{e}\right]}+\left(x_{1} \cdots x_{m}\right)^{p^{e}-1}$.

ii) $\left(I^{\left[p^{e}\right]}:_{S} I\right)=I^{\left[p^{e}\right]}+J_{p^{e}}+\left(x_{1} \cdots x_{m}\right)^{p^{e}-1}$. 
where $J_{p^{e}}$ is generated by monomials $\mathbf{x}^{\gamma}:=x_{1}^{c_{1}} \cdots x_{m}^{c_{m}}$ satisfying $c_{i} \in\left\{0, p^{e}-1, p^{e}\right\}$. These monomials are not contained in $I^{\left[p^{e}\right]}+\left(x_{1} \cdots x_{m}\right)^{p^{e}-1}$ whenever $c_{i}=p^{e}, c_{j}=p^{e}-1, c_{k}=0$ for $1 \leq i, j, k \leq m$.

As an immediate consequence we have the following result.

Theorem 4.1. [1, Theorem 3.5] The Frobenius algebra $\mathcal{F}\left(E_{R}\right)$ associated to a StanleyReisner ring $R$ is either principally generated or infinitely generated.

More interestingly, the formula obtained for $\left(I^{\left[p^{e}\right]}:_{S} I\right)$ is exactly the same $\forall e$, so one only has to compute the first graded piece $\mathcal{F}^{1}\left(E_{R}\right) \cong\left(I^{[p]}:_{S} I\right)$ in order to describe the whole Frobenius algebra $\mathcal{F}\left(E_{R}\right)$. When $\mathcal{F}\left(E_{R}\right)$ is principally generated it is generated by $\left(x_{1} \cdots x_{m}\right)^{p-1}$. On the other hand, when it is infinitely generated, we have that $\mathcal{F}^{e}\left(E_{R}\right)$ has $\mu$ minimal monomial generators corresponding to the minimal generators of $J_{p^{e}}$ plus the generator $\left(x_{1} \cdots x_{m}\right)^{p^{e}-1}$. A. F. Boix and S. Zarzuela [4, Theorem 2.15] gave a nice interpretation of the monomial generators in terms of the maximal free pairs of the simplicial complex associated to the Stanley-Reisner ring $R$.

A full description of the complexity sequence is still an open question. A. F. Boix and S. Zarzuela proved in [4, Theorem 3.8] that the generators coming from monomials $\mathbf{x}^{\gamma} \in J_{p^{e}}$ with minimal support ${ }^{1}$ are new, that is they cannot be obtained from the $R$ algebra generated by $\mathcal{F}^{0}\left(E_{R}\right), \ldots, \mathcal{F}^{e-1}\left(E_{R}\right)$. Then, they conclude that each piece $\mathcal{F}^{e}\left(E_{R}\right)$ adds at most $\mu$ new generators. In the case that all the monomials in $J_{p^{e}}$ have minimal support we have that the complexity sequence is

$$
\left\{c_{e}\right\}_{e \geq 0}=\{1, \mu+1, \mu, \mu, \mu, \ldots\}
$$

so we have the linear recurrence $c_{e+1}-c_{e}=0$ for all $e \geq 2$. In particular, its generating function is

$$
\mathcal{G}_{\mathcal{F}\left(E_{R}\right)}(T)=1+(\mu+1) T+\sum_{e \geq 2} \mu T^{e}=1+(\mu+1) T+T^{2} \frac{\mu}{1-T}=\frac{1+\mu T-T^{2}}{1-T} .
$$

Example 4.2. Let $I=(x, y) \cap(z, w)$ be a squarefree monomial ideal in $S=k[[x, y, z, w]]$. Then we have

$$
\frac{\left(I^{\left[p^{e}\right]}:_{S} I\right)}{I^{\left[p^{e}\right]}}=\left(x^{p^{e}-1} y^{p^{e}-1} z^{p^{e}}, x^{p^{e}} z^{p^{e}-1} w^{p^{e}-1}, y^{p^{e}} z^{p^{e}-1} w^{p^{e}-1}, x^{p^{e}-1} y^{p^{e}-1} w^{p^{e}}\right)+(x y z w)^{p^{e}-1}
$$

and the generating function is

$$
\mathcal{G}_{\mathcal{F}\left(E_{R}\right)}(T)=\frac{1+4 T-T^{2}}{1-T} .
$$

\footnotetext{
$\overline{1^{1} \text { The support }}$ of a monomial $\mathbf{x}^{\gamma}:=x_{1}^{c_{1}} \cdots x_{n}^{c_{n}}$ is $\operatorname{supp}\left(\mathbf{x}^{\gamma}\right):=\left\{i \in\{1, \ldots, n\} \mid c_{i} \neq 0\right\}$.
} 
4.2. Veronese subrings. Let $R=k\left[x_{1}, \ldots, x_{m}\right]$ be the polynomial ring over a field of characteristic $p>0$ that we consider as a $\mathbb{N}$-graded ring $R=\bigoplus_{k \geq 0} R_{k}$. Given an integer $r \geq 1$, the $r$-th Veronese subring of $R$ is

$$
V_{r}(R)=\bigoplus_{k \geq 0} R_{r k}
$$

M. Katzman, K. Schwede, A. Singh and W. Zhang gave a complete description of the Frobenius algebra $\mathcal{F}\left(E_{R}\right)$ in [12, Proposition 4.3] using the T-construction.

Proposition 4.3. Under the previous assumptions, $\mathcal{F}^{e}\left(E_{R}\right)$ is the left $R$-module generated by the elements

$$
\frac{1}{x_{1}^{\alpha_{1}} \cdots x_{d}^{\alpha_{d}}} F^{e}
$$

where $F^{e}$ is the e-th Frobenius map, $\alpha_{k} \leq p^{e}-1$ for each $k$, and $\sum \alpha_{k} \equiv 0 \bmod n$.

In [12, Example 4.5] they consider the case where $m=2$ and $r=3$. The Frobenius algebra $\mathcal{F}\left(E_{R}\right)$ is infinitely generated when the characteristic of the field $k$ is $p=3$. A straightforward computation shows that for $m=2$ and $r=p$, the complexity sequence is $\left\{c_{e}\right\}_{e \geq 0}=\{1, p-1, p-1, p-1, \ldots\}$ so the generating function is

$$
\mathcal{G}_{\mathcal{F}\left(E_{R}\right)}(T)=1+\sum_{e \geq 1}(p-1) T^{e}=1+T \frac{(p-1)}{1-T}=\frac{1+(p-2) T}{1-T} .
$$

The complexity of the Frobenius algebra is $\operatorname{cx}\left(\mathcal{F}\left(E_{R}\right)\right)=1$ and the Frobenius complexity is $\operatorname{cx}_{F}(R)=0$.

4.3. Determinantal rings. Let $X$ be an $n \times m$ generic matrix with $m>n \geq 2$ and $I:=I_{2}(X)$ the ideal generated by the $2 \times 2$ minors of $X$. In this subsection we will compute the generating function of the Frobenius algebra $\mathcal{F}\left(E_{R}\right)$, where $R$ is the completion of the determinantal ring $k[X] / I$.

A precise description of the Frobenius algebra for the case of a $2 \times 3$ generic matrix using Fedder's approach was given in [12] so, from this construction one may extract its complexity sequence. However, F. Enescu and Y. Yao took a different approach in [6] and [7] to describe the complexity sequence of $\mathcal{F}\left(E_{R}\right)$ in the general case of a $n \times m$ generic matrix. Actually, they proved in [7, Theorem 1.20] that the complexity sequence of $\mathcal{F}\left(E_{R}\right)$ coincides with the complexity sequence of the $\mathbb{N}$-graded ring $A=T\left(V_{m-n}\left(k\left[x_{1}, \ldots, x_{m}\right]\right)\right)$, which is the twisted ring associated to the $(m-n)$-th Veronese subring of a polynomial ring $k\left[x_{1}, \ldots, x_{m}\right]$ in $m$ variables.

Next we are going to describe this complexity sequence in the general framework considered in [6]. Namely, they consider the twisted ring $T\left(V_{r}\left(R\left[x_{1}, \ldots, x_{m}\right]\right)\right)$, where $R$ is any commutative ring $R$ of characteristic $p>0$ not only a field. In [6, Proposition 3.1] they give a precise description but, for our purposes, we will consider the interpretation they provide in [7, Discussion 3.2] that we briefly describe. 
Set

$$
M_{p, m}(k):=\operatorname{rank}_{R}\left(R\left[x_{1}, \ldots, x_{m}\right] /\left(x_{1}^{p}, \ldots, x_{m}^{p}\right)\right)_{k} .
$$

These positive integers can be read off as the coefficients of the following polynomial

$$
\sum_{k=0}^{m(p-1)} M_{p, m}(k) T^{k}=\left(1+T+\cdots+T^{p-1}\right)^{m}
$$

In particular $M_{p, m}(k)=0$ for $k<0$ and $k>m(p-1)$. Moreover they satisfy the symmetric property $M_{p, m}(k)=M_{p, m}(m(p-1)-k)=0$.

Now we construct the $(m-r-1) \times(m-r-1)$ matrix

$$
U:=\left(\begin{array}{ccc}
M_{p, m}(r(p-1)+p-1) & \cdots & M_{p, m}(r(p-1)+p-(m-r-1)) \\
\vdots & \ddots & \vdots \\
M_{p, m}(r(p-1)+(m-r-1) p-1) & \cdots & M_{p, m}(r(p-1)+(m-r-1) p-(m-r-1))
\end{array}\right)
$$

that is, $U=\left(u_{i j}\right)$ with $u_{i j}:=M_{p, m}(r(p-1)+i p-j)$.

Consider the discrete dynamical system $X_{e}=U X_{e-1}$, or equivalently $X_{e}=U^{e} X_{0}$, with the initial conditions

$$
X_{0}=\left(M_{p, m}(r(p-1)+p), M_{p, m}(r(p-1)+2 p), \ldots, M_{p, m}(r(p-1)+(m-r-1) p)\right)^{\top} .
$$

The complexity sequence of $T\left(V_{r}\left(R\left[x_{1}, \ldots, x_{m}\right]\right)\right)$ for any positive integers $r, m$ such that $r+1<m$ is:

$$
\begin{aligned}
& \cdot c_{0}=1 . \\
& \cdot c_{1}=\operatorname{rank}_{R}\left(R\left[x_{1}, \ldots, x_{m}\right]\right)_{r(p-1)}=\left(\begin{array}{c}
r(p-1)+(m-1) \\
m-1
\end{array}\right) . \\
& \cdot c_{e}=Y \cdot X_{e-2}, \text { for } e \geq 2, \text { where: } \\
Y= & \left(\left(\begin{array}{c}
r(p-1)-1+m-1 \\
m-1
\end{array}\right),\left(\begin{array}{c}
r(p-1)-2+m-1 \\
m-1
\end{array}\right), \ldots,\left(\begin{array}{c}
r(p-1)-(m-r-1)+m-1 \\
m-1
\end{array}\right)\right) .
\end{aligned}
$$

The main result of this subsection is the following result that describes the linear recurrence of this complexity sequence.

Theorem 4.4. Let $m(T)=T^{s}+a_{1} T^{s-1}+\cdots+a_{s}$ be the minimal polynomial of the matrix $U$ associated to the twisted Veronese subring $A=T\left(V_{r}\left(R\left[x_{1}, \ldots, x_{m}\right]\right)\right)$. Then, the complexity sequence of $\left\{c_{e}(A)\right\}_{e} \geq 0$ satisfies the linear recurrence

$$
c_{e+s}+a_{1} c_{e+s-1}+\cdots+a_{s} c_{e}=0,
$$

for all $e \geq 2$.

Proof. We start with the case where the matrix $U$ diagonalizes. Let $v_{1}, \ldots, v_{m-r-1}$ be the set of eigenvectors of $U$ with eigenvalues $\lambda_{1}, \ldots, \lambda_{s}$ with $s \leq m-r-1$. In particular, if the characteristic polynomial of $U$ is $p(T)=\left(T-\lambda_{1}\right)^{m_{1}} \cdots\left(T-\lambda_{s}\right)^{m_{s}}$, then the minimal 
polynomial is $m(T)=\left(T-\lambda_{1}\right) \cdots\left(T-\lambda_{s}\right)$. Let $S$ be the change basis matrix such that $U=S D S^{-1}$ with $D$ being the diagonal matrix with the set of eigenvalues as entries. Thus we have:

$$
X_{e}=U^{e} X_{0}=S D^{e} S^{-1} X_{0}=\alpha_{1} \lambda_{1}^{e} v_{1}+\cdots+\alpha_{m-r-1} \lambda_{s}^{e} v_{m-r-1},
$$

where $S^{-1} X_{0}=\left(\alpha_{1}, \ldots, \alpha_{m-r-1}\right)^{\top}$. Therefore, the elements of the complexity sequence are

$$
c_{e}=Y \cdot X_{e-2}=\left(\alpha_{1} \lambda_{1}^{e-2}\right) Y \cdot v_{1}+\cdots+\left(\alpha_{m-r-1} \lambda_{s}^{e-2}\right) Y \cdot v_{m-r-1} .
$$

Recall that the $\mathcal{Z}$-transform of the sequence $\left\{\lambda^{e}\right\}_{e \geq 0}$ is

$$
\mathscr{Z}\left[\lambda^{e}\right](Z)=\frac{Z}{Z-\lambda} \quad, \quad \mathscr{Z}\left[\lambda^{e}\right]\left(\frac{1}{T}\right)=\frac{1}{1-\lambda T} .
$$

It follows that

$$
\sum_{e \geq 2} c_{e} T^{e-2}=\frac{\alpha_{1} Y \cdot v_{1}}{1-\lambda_{1} T}+\cdots+\frac{\alpha_{m-r-1} Y \cdot v_{m-r-1}}{1-\lambda_{s} T}=\frac{P(T)}{\left(1-\lambda_{1} T\right) \cdots\left(1-\lambda_{s} T\right)}
$$

with $\operatorname{deg}(P(T))<s$ and therefore

$$
\mathcal{G}_{A}(T)=c_{0}+c_{1} T+T^{2} \sum_{e \geq 2} c_{e} T^{e-2}=c_{0}+c_{1} T+T^{2} \frac{P(T)}{\left(1-\lambda_{1} T\right) \cdots\left(1-\lambda_{s} T\right)}
$$

and the result follows from Proposition 2.4.

If the matrix $U$ does not diagonalize, we may play the same game with the corresponding Jordan normal form. Let $m(T)=\left(T-\lambda_{1}\right)^{j_{1}} \cdots\left(T-\lambda_{s}\right)^{j_{s}}$ be the minimal polynomial of $U$ and $S$ the change basis matrix such that $U=S J S^{-1}$ with $J$ being the normal Jordan form.

To avoid heavy notation, we will start developing carefully the case where $J$ is a $j \times j$ Jordan block corresponding to an eigenvalue $\lambda$. Notice that we are assuming that the characteristic and the minimal polynomial are both $(T-\lambda)^{j}$. Let $v_{1}, \ldots, v_{j}$ be the set of generalized eigenvectors. Then

$$
\begin{aligned}
X_{e} & =U^{e} X_{0}=S J^{e} S^{-1} X_{0}=\alpha_{1}\left[\lambda^{e} v_{1}+\left(\begin{array}{c}
e \\
1
\end{array}\right) \lambda^{e-1} v_{2}+\cdots+\left(\begin{array}{c}
e \\
j-1
\end{array}\right) \lambda^{e-(j-1)} v_{j}\right]+ \\
& +\alpha_{2}\left[\lambda^{e} v_{2}+\cdots+\left(\begin{array}{c}
e \\
j-2
\end{array}\right) \lambda^{e-(j-2)} v_{j}\right]+\cdots+\alpha_{j}\left[\lambda^{e} v_{j}\right]
\end{aligned}
$$

where $S^{-1} X_{0}=\left(\alpha_{1}, \ldots, \alpha_{j}\right)^{\top}$. Therefore, we have

$$
\begin{aligned}
c_{e} & =Y \cdot X_{e-2}= \\
& =\left(\alpha_{1} \lambda^{e-2}\right) Y \cdot v_{1}+\left(\alpha_{1}\left(\begin{array}{c}
e-2 \\
1
\end{array}\right) \lambda^{e-3}\right) Y \cdot v_{2}+\cdots+\left(\alpha_{1}\left(\begin{array}{c}
e-2 \\
j-1
\end{array}\right) \lambda^{e-2-(j-1)}\right) Y \cdot v_{j}+ \\
& +\left(\alpha_{2} \lambda^{e-2}\right) Y \cdot v_{2}++\cdots+\left(\alpha_{2}\left(\begin{array}{c}
e-2 \\
j-2
\end{array}\right) \lambda^{e-2-(j-2)}\right) Y \cdot v_{j}+\cdots+\left(\alpha_{j} \lambda^{e-2}\right) Y \cdot v_{j} .
\end{aligned}
$$


The $\mathcal{Z}$-transform of the sequence $\left\{\left(\begin{array}{l}e \\ i\end{array}\right) \lambda^{e-i}\right\}_{e \geq 0}$ is

$$
\mathscr{Z}\left[\left(\begin{array}{l}
e \\
i
\end{array}\right) \lambda^{e-i}\right](Z)=\frac{Z}{(Z-\lambda)^{i+1}} \quad, \quad \mathscr{Z}\left[\left(\begin{array}{l}
e \\
i
\end{array}\right) \lambda^{e-i}\right]\left(\frac{1}{T}\right)=\frac{T^{i}}{(1-\lambda T)^{i+1}} .
$$

Thus we have

$$
\begin{aligned}
\sum_{e \geq 2} c_{e} T^{e-2} & =\frac{\alpha_{1}\left(Y \cdot v_{1}\right)}{1-\lambda T}+\frac{\alpha_{1}\left(Y \cdot v_{2}\right) T}{(1-\lambda T)^{2}}+\cdots+\frac{\alpha_{1}\left(Y \cdot v_{j}\right) T^{j-1}}{(1-\lambda T)^{j}}+ \\
& +\frac{\alpha_{2}\left(Y \cdot v_{2}\right)}{1-\lambda T}+\cdots+\frac{\alpha_{2}\left(Y \cdot v_{j}\right) T^{j-2}}{(1-\lambda T)^{j-1}}+\cdots+\frac{\alpha_{j}\left(Y \cdot v_{j}\right)}{1-\lambda T}= \\
& =\frac{P_{\lambda}(T)}{(1-\lambda T)^{j}},
\end{aligned}
$$

where $P_{\lambda}(T)$ is a polynomial with $\operatorname{deg}\left(P_{\lambda}(T)\right)<j$.

In the general case we will get a rational function of the form $\frac{P_{\lambda}(T)}{(1-\lambda T)^{j}}$ for each Jordan block of size $j$. Since the maximum size of the Jordan blocks associated to an eigenvalue is the algebraic multiplicity of this eigenvalue as a root of the minimal polynomial we get

$$
\mathcal{G}_{A}(T)=c_{0}+c_{1} T+T^{2} \sum_{e \geq 2} c_{e} T^{e-2}=c_{0}+c_{1} T+T^{2} \frac{P(T)}{\left(1-\lambda_{1} T\right)^{j_{1}} \cdots\left(1-\lambda_{s} T\right)^{j_{s}}}
$$

with $\operatorname{deg}(P(T))<j_{1}+\cdots+j_{s}$ and the result follows from Proposition 2.4

Remark 4.5. Numerical experimentation with MATLAB [14] suggests that all the eigenvalues of the matrix $U$ are different (albeit we may have complex eigenvalues). Thus the matrix $U$ diagonalizes and the characteristic and minimal polynomial coincide. In particular, the linear recurrence that the complexity sequence satisfies is given by the characteristic polynomial.

Example 4.6. Consider the ring $A=T\left(V_{1}\left(R\left[x_{1}, \ldots, x_{18}\right]\right)\right)$, where $R$ is a ring of characteristic $p=31$. Then, the set of eigenvalues of the corresponding matrix $U$ is

$$
\begin{aligned}
& Z=1.0 e+25 * \\
& 2.255011677416268+0.000000000000000 i \\
& 0.072742312174691+0.000000000000000 i \\
& 0.002346526199128+0.000000000000000 i \\
& 0.000075694393453+0.000000000000000 i \\
& 0.000002441754572+0.000000000000000 i \\
& 0.000000078766244+0.000000000000000 i \\
& 0.000000002540832+0.000000000000000 i \\
& 0.000000000081958+0.000000000000000 i \\
& 0.000000000002643+0.000000000000000 i
\end{aligned}
$$




$$
\begin{array}{r}
0.000000000000085+0.000000000000000 i \\
0.000000000000003+0.000000000000000 i \\
0.000000000000000+0.000000000000000 i \\
-0.000000000000000+0.000000000000000 i \\
-0.000000000000000-0.000000000000000 i \\
0.000000000000000+0.000000000000000 i \\
0.000000000000000+0.000000000000000 i
\end{array}
$$

If we take a close look we can check that there exist complex eigenvalues:

$\gg Z(13) \quad$ ans $=-2.634600279958723 e+08+2.351626510499541 e+08 i$
$>Z(14) \quad$ ans $=-2.634600279958723 e+08-2.351626510499541 e+08 i$

All the entries of the matrix $U$ are positive real numbers. Thus, the Perron-Frobenius theorem says that it has a unique eigenvalue with maximal absolute value that we simply denote as Perron-Frobenius eigenvalue. Using Theorem 2.5 we can deduce the complexity and the Frobenius complexity of the Frobenius algebra. This was already observed by F. Enescu and Y. Yao in [7].

Corollary 4.7. Let $\lambda$ be the Perron-Frobenius eigenvalue of the matrix $U$ associated to the twisted ring $A=T\left(V_{r}\left(R\left[x_{1}, \ldots, x_{m}\right]\right)\right)$. Then $\operatorname{cx}(A)=\lambda$.

We end this section with some computations for the twisted Veronese subring $A=$ $T\left(V_{r}\left(R\left[x_{1}, \ldots, x_{m}\right]\right)\right)$ with $R$ being of characteristic $p$. F. Enescu and Y. Yao computed explicitly the case $(m, r, p)=(4,1,2)$ in [6] and $(m, r, p)=(5,2,3)$ in [7]. In the following tables we present, for some small cases, the minimal polynomial $m(T)$, the complexity and the Frobenius complexity of $A$ varying the characteristic of the ring. We point out that $\lim _{p \rightarrow \infty} \operatorname{cx}_{F}(A)=m-1($ see $[7$, Theorem 4.1]).

\begin{tabular}{|c|c|c|c|}
\hline$p$ & $m(T)$ & $\operatorname{cx}(A)$ & $\operatorname{cx}_{F}(A)$ \\
\hline \hline 2 & $T^{3}-25 T^{2}+165 T-280$ & 15.5436 & 3.9583 \\
\hline 3 & $T^{3}-105 T^{2}+2205 T-8505$ & 78.1909 & 3.9679 \\
\hline 5 & $T^{3}-710 T^{2}+66625 T-687500$ & 601.0565 & 3.9757 \\
\hline 7 & $T^{3}-2590 T^{2}+660275 T-12941390$ & 2306.1190 & 3.9793 \\
\hline 11 & $T^{3}-15103 T^{2}+14857953 T-692680351$ & 14048.9228 & 3.9828 \\
\hline 13 & $T^{3}-29120 T^{2}+47246485 T-3040889670$ & 27399.7078 & 3.9838 \\
\hline
\end{tabular}

TABLE 1 . The case $(m, r)=(5,1)$. 


\begin{tabular}{|c|c|c|c|}
\hline$p$ & $m(T)$ & $\mathrm{cx}(A)$ & $\mathrm{cx}_{F}(A)$ \\
\hline \hline 2 & $T^{2}-15 T+40$ & 11.5311 & 3.5275 \\
\hline 3 & $T^{2}-60 T+420$ & 51.9089 & 3.5950 \\
\hline 5 & $T^{2}-390 T+9625$ & 363.5230 & 3.6633 \\
\hline 7 & $T^{2}-1400 T+82320$ & 1338.4982 & 3.6997 \\
\hline 11 & $T^{2}-8052 T+1591876$ & 7849.1923 & 3.7400 \\
\hline 13 & $T^{2}-15470 T+4844385$ & 15150.2437 & 3.7528 \\
\hline
\end{tabular}

TABle 2. The case $(m, r)=(5,2)$.

In order to describe the generating function we only have to follow the arguments developed in the proof of Theorem 4.4. For example, in the case that $(m, r)=(5,1)$ and $p=5$ we have:

$$
\mathcal{G}_{A}(T)=1+70 T+T^{2}\left(\frac{15575-2913750 T+38359375 T^{2}}{1-710 T+66625 T^{2}-687500 T^{3}}\right)
$$

\section{Open questions}

Let $R$ be a commutative Noetherian ring and let $A=\oplus_{e \geq 0} A_{e}$ be a (non-necessarily commutative) $\mathbb{N}$-graded ring. When dealing with the generating function of $A$ and its relation with the complexity, there are some questions that immediately come to mind. The obvious one is:

- When is the generating function of $A$ a rational function?

In this case, as a consequence of Theorem 2.5, we have that the complexity of $A$ is finite. A priori, rationality of the generating function seems to be a stronger condition that involves the structure of the complexity sequence rather than its asymptotic behaviour. Then we may ask:

- Is there any $\mathbb{N}$-graded ring $A$ with finite complexity but non-rational generating function?

Assume that the generating sequence of $A$ is rational, then we may consider a generalization of the complexity $\operatorname{cx}(A)$ just taking into account all the eigenvalues of the characteristic polynomial associated to the corresponding linear recurrence ordered by their absolute values. Namely, we may consider the complexity eigenvalues of $A$

$$
\operatorname{cx}_{\mathrm{eig}}(A)=\left(\left|\lambda_{1}\right|,\left|\lambda_{2}\right|, \ldots,\left|\lambda_{k}\right|\right),
$$

where the largest eigenvalue corresponds to the complexity (see Corollary 2.8). In the case that $R$ is a local complete commutative ring of positive characteristic $p>0$, we may 
also consider the Frobenius complexity eigenvalues

$$
\mathrm{cx}_{F, \mathrm{eig}}(R):=\log _{p}\left(\mathrm{cx}_{\mathrm{eig}}\left(\mathcal{F}\left(E_{R}\right)\right)\right.
$$

Then we may ask:

- Does $\lim _{p \rightarrow \infty} \operatorname{cx}_{F, \text { eig }}(R)$ exist?

- What is this limit for the twisted Veronese subring $A=T\left(V_{r}\left(R\left[x_{1}, \ldots, x_{m}\right]\right)\right)$ ?

Numerical experimentation with the twisted Veronese subring suggested the following question:

- Are all the eigenvalues of the matrix $U$ different so $U$ diagonalizes?

Finally, it would be really useful to have a larger set of examples, not only new examples of Frobenius algebras but other possible interesting objects such as rings of differential operators over non-regular rings in any characteristic.

\section{REFERENCES}

[1] J. Àlvarez Montaner, A. F. Boix and S. Zarzuela, Frobenius and Cartier algebras of Stanley-Reisner rings, J. Algebra 358 (2012), 162-177.

[2] M. Blickle, The intersection homology D-module in finite characteristic, Ph.D. thesis, (2001), arXiv:0110244.

[3] M. Blickle, Test ideals via algebras of p -linear maps, J. Algebraic Geom., 22 (2013), 49-83.

[4] A. F. Boix and S. Zarzuela, Frobenius and Cartier algebras of Stanley-Reisner rings (II), Acta Math Vietnam (2019). https://doi.org/10.1007/s40306-018-00314-1.

[5] S. N. Elaydi, An introduction to difference equations. Undergraduate Texts in Mathematics. SpringerVerlag, New York, 1996. xiv+389 pp.

[6] F. Enescu and Y. Yao, The Frobenius complexity of a local ring of prime characteristic, J. Algebra 459 (2016), 133-156.

[7] F. Enescu and Y. Yao, On the Frobenius complexity of determinantal ideals, J. Pure Appl. Algebra 222 (2018), 414-432.

[8] R. Fedder. F-purity and rational singularity, Trans. Amer. Math. Soc., 278 (1983), 461-480.

[9] M. Hochster and C. Huneke. Tight closure, invariant theory, and the Briançon-Skoda theorem, J. Amer. Math. Soc. 3 (1990), 31-116.

[10] M. Katzman, Parameter-test ideals of Cohen-Macaulay rings, Compos. Math. 144 (2008), 933-948.

[11] M. Katzman, A non-finitely generated algebra of Frobenius maps, Proc. Amer. Math. Soc. 138 (2010), 2381-2383.

[12] M. Katzman, K. Schwede, A. Singh and W. Zhang, Rings of Frobenius operators, Math. Proc. Cambridge Philos. Soc. 157 (2014), 151-167.

[13] G. Lyubeznik and K. Smith, On the commutation of the test ideal with localization and completion, Trans. Amer. Math. Soc. 353 (2001), 3149-3180.

[14] MATLAB version 8.5.0. Natick, Massachusetts: The MathWorks Inc., 2015.

[15] J. Page, The Frobenius complexity of Hibi rings, J. Pure Appl. Algebra 223 (2019), 580-604

[16] K. Schwede, Test ideals in non- $\mathbb{Q}$-Gorenstein rings, Trans. Amer. Math. Soc. 363 (2011), 5925-5941.

Departament de Matemàtiques, Universitat Politècnica de Catalunya, Av. Diagonal 647, BARCELONA 08028, Spain

E-mail address: Josep.Alvarez@upc.edu 